Angelika SCHOBER

\title{
KULTURELLE BILDUNG IN DEUTSCHLAND UND IN FRANKREICH
}

In seinem einundzwanzigsten Brief über die ästhetische Erziehung des Menschen bezeichnet Friedrich Schiller die Schönheit als unsere zweite Schöpferin: „Es ist also nicht bloß $\beta$ poetisch erlaubt, sondern auch philosophisch richtig, wenn man die Schönheit unsere zweite Schöpferin nennt." ${ }^{\text {"I }}$ Und er erachtet diese Neuschöpfung des Menschen durch die Kunst für notwendig in politischer Hinsicht, also in Hinblick auf die Errichtung eines humanen Staates. Im zweiten Brief lesen wir, „da $\beta$ man, um jenes politische Problem zu lösen, durch das ästhetische den Weg nehmen muß, weil es die Schönheit ist, durch welche man zur Freiheit wandert. “2

Schiller verfasste seine Briefe über die ästhetische Erziehung des Menschen in den Jahren 1793 - 1794 als Antwort auf die Schreckensherrschaft der Französischen Revolution und die Hinrichtung von Ludwig XVI. Damit nicht Gewalt die Politik bestimmt, fordert er Erziehung. Jeder Einzelne ist zu bilden und zwar auf ästhetische Weise, also durch Kunst. Somit ist kulturelle Bildung ein politischer Auftrag, sie stellt einen wesentlichen Faktor der politischen Bildung dar. Und heute noch können wir Nachklänge dieser Überzeugung in Deutschland finden, in der bildungspolitischen Theorie gleichermaßen wie in der pädagogischen Praxis. Doch wie sieht es in Frankreich aus? ${ }^{3}$

$1 \quad$ Briefe über die ästhetische Erziehung des Menschen. In Friedrich Schiller, Ausgewählte Werke, hrsg. und eingeleitet von Ernst Müller, Bd.5 Schriften zum Theater, zur Philosophie der Ästhetik und der Geschichte, Kohlhammer Verlag Stuttgart, 1955, S. 336.

2 Ibid., S.263. Siehe hierzu meinen Aufsatz ,Schillers Briefe über die ästhetische Erziehung des Menschen als Antwort auf die Französische Revolution und ihre Folgen“, Kairoer Germanistische Studien n²0, 2012-2013, S.213-233.

3 Die folgenden Beobachtungen stellen keine systematische Untersuchung dar. Sie möchten dazu anregen, über die Problematik nachzudenken und skizzieren einige Aspekte anhand von aktuellen Verlautbarungen der zuständigen Ministerien in Deutschland und Frankreich.

Kairoer Germanistische Studien 21 (2014) 


\section{Bildung als politische Priorität}

Sowohl in Deutschland als auch in Frankreich wird heute die Bedeutung von Bildung auf höchster politischer Ebene ausdrücklich betont. Auf dem Bildungsgipfel am 22. Oktober 2008 in Dresden beschlossen Bund und Länder die sogenannte Qualifikationsinitiative „Aufstieg durch Bildung“. ${ }^{4}$ Und Punkt 1 des Berichtes zur Umsetzung dieser Initiative aus dem Jahre 2012 fordert, dass „Bildung [...] in Deutschland höchste Priorität haben“ soll'. In Frankreich steht das Bildungsthema ebenfalls an zentraler Stelle. Seit Staatspräsident François Hollande 2012 zur Reform des gesamten Bildungssektors aufrief unter dem Motto „Refondation de 1'Ecole“ - womit eine grundlegende Umstrukturierung des gesamten Schul- und Bildungswesens gemeint ist. Auf diese Weise soll die sogenannte ,,promesse républicaine de réussite pour tous" eingelöst werden - das republikanische Versprechen eines sozialen Aufstiegs für alle. Diese französische Richtlinie entspricht in vieler Hinsicht der deutschen Qualifikationsinitiative aus dem Jahre 2009, welche bezeichnenderweise „Aufstieg durch Bildung“ benannt wurde. Umso mehr als der Umsetzungsbericht von 2012 fordert, alle Kinder zu fördern: ,Jede und jeder soll die Chance zum Aufstieg durch Bildung

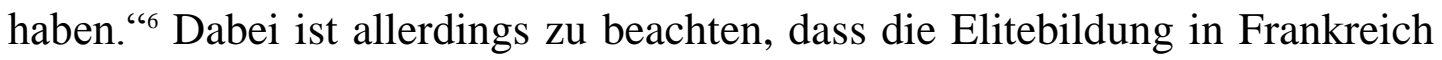
diskreter angesprochen wird als in Deutschland. Das französische Programm bezüglich der „Refondation de l'Ecole“ erwähnt sie nicht eigens, anders als der deutsche Bericht zur Umsetzung der Qualifikationsinitiative, welcher

\footnotetext{
$4 \quad$ Seit 2009 veröffentlichen die Regierungschefs von Bund und Ländern jährlich einen gemeinsamen Bericht über die Umsetzung der beschlossenen Maßnahmen. Aufstieg durch Bildung, Die Qualifizierungsinitiative für Deutschland, Bericht zur Umsetzung 2012, Sekretariat der Ständigen Konferenz der Kultusminister der Länder in der Bundesrepublik Deutschland; Gemeinsame Wissenschaftskonferenz (GWK), Beschluss der KMK vom 18.10.2012, Beschluss der GWK vom 16.11.2012. Abrufbar unter: www.kmk.org sowie www.gwk-bonn.de und www.bmbf.de.

5 Aufstieg durch Bildung, Die Qualifizierungsinitiative für Deutschland, Bericht zur Umsetzung 2012, Überschrift von Kapitel 1 des ersten Handlungsfeldes, S.2

6 Ibid., Punkt 4.2. lautet: ,Jedes Kind soll bestmögliche Startbedingungen haben“ und Punkt 3: „Jede und jeder soll einen Schul- und Berufsabschluss schaffen können“.
} 
ausdrücklich feststellt: „Spitzen- und Breitenförderung sind zwei Seiten einer Medaille".?

\section{Stellenwert und Begründung ästhetisch-künstlerisch-kultureller Bildung}

Indem in Deutschland wie in Frankreich die Bedeutung der ästhetischkünstlerisch-kulturellen Erziehung betont wird, respektieren beide Länder, dass die UNESCO kulturelle Bildung als ein Menschenrecht definiert, welches dazu „befähigen soll, den eigenen kulturellen Interessen $\mathrm{zu}$ folgen, künstlerisch-ästhetische Wahrnehmung und Urteilsvermögen zu entwickeln und am kulturellen Leben teilzunehmen. “8 Übereinstimmung besteht zudem darin, dass beide Staaten kulturelle Bildung auf allen Stufen fordern. In Frankreich erörtert das Erziehungsministerium diesbezügliche Fragen unter der Überschrift „De la maternelle au baccalauréat - l'éducation artistique et culturelle" (Vom Kindergarten bis zum Abitur - künstlerische und kulturelle Bildung $)^{9}$. Auf deutscher Seite geht dies zum Beispiel aus Folgendem hervor: das Vorwort der vom Bayerischen Staatsministerium für Unterricht und Kultus herausgegebenen „Handreichung“ zum Thema „Ästhetische Bildung“ trägt den Titel „,̈̈sthetische Bildung - ein Konzept nicht nur für Gymnasien“. ${ }^{10}$ Und der Bildungsbericht der Bundesregierung aus dem Jahre 2012 unterstreicht, dass „kulturelle/musisch-ästhetische Bildung“ eine „wichtige

Ibid., S.24.

UNESCO, Leitfaden für kulturelle Bildung. Schaffung kreativer Kapazitäten für das 21. Jahrhundert, Paris, 2006. zitiert nach dem Bildungsbericht der Bundesregierung: Autorengruppe Bildungsberichterstattung, Bildung in Deutschland 2012. Ein indikatorengestützter Bericht mit einer Analyse zur kulturellen Bildung im Lebenslauf, gefördert mit Mitteln der Ständigen Konferenz der Kultusminister der Länder in der Bundesrepublik Deutschland und des Bundesministeriums für Bildung und Forschung, hrsg. Bundesministerium für Bildung und Forschung, W. Bertelsmann Verlag, Bielefeld 2012, (360S.), S.157, Fußnote 4.

9 Siehe website des Ministère de l'éducation nationale, eingesehen am 4.6. 2013.

Stefan Krimm, „Ästhetische Bildung - ein Konzept nicht nur für Gymnasien“, in Bayerisches Staatsministerium für Unterricht und Kultus, Ästhetische Bildung. Handreichung, hrsg. vom Staatsinstitut für Schulqualität und Bildungsforschung, München, sd. 
Voraussetzung für autonome und kritische Teilhabe an Gesellschaft und Politik“ ist. $^{11}$

In Frankreich, wo man lieber von kultureller beziehungsweise künstlerisch-kultureller als von ästhetischer Bildung spricht, gehört sie zu den fünf Prioritäten des Rundschreibens zur Vorbereitung des Schuljahres 20132014, welches am 11.Aprril 2013 vom Erziehungsministerium veröffentlicht wurde. Zwar steht sie nur an letzter Stelle, doch Punkt 5 des ersten Teils trägt die Überschrift Développer l'éducation artistique et culturelle (EAC), was bedeutet, dass die künstlerische und kulturelle Bildung zu entwickeln ist. Als Begründung gilt vor allem, dass sie für die Demokratisierung der Gesellschaft und für Chancengleichheit unentbehrlich ist. ${ }^{12}$ Eine gemeinsame Erklärung der zwei zuständigen Ministerien ${ }^{13}$ unterstreicht zudem am 21. November 2012 ihre Wichtigkeit: „Die Regierung verfolgt das Ziel, allen Jugendlichen in allen Teilen des Landes Zugang $\mathrm{zu}$ Kunst und Kultur zu ermöglichen, vom Kleinkindalter bis zur Universität“. Dies soll auf drei Ebenen geschehen: durch theoretischen Unterricht (wobei Kunstgeschichte eine große Rolle spielt), durch selbständige künstlerische Praxis der Schüler und mit Hilfe von Begegnungen mit Kunstwerken und Künstlern. ${ }^{14}$

In Deutschland betont der Bildungsbericht der Bundesregierung aus dem Jahre 2012, dass ,kulturelle/musisch-ästhetische Bildung eigenständige Ziele hat" und versteht sie als ,eine kulturelle Praxis, die dazu beiträgt, Individuen zu einem selbstbestimmten Leben, zur Entdeckung und Entfaltung ihrer expressiven Bedürfnisse sowie zur aktiven Teilnahme an Kultur zu

11 Bildungsbericht der Bundesregierung, Kulturellel musisch-ästhetische Bildung im Lebenslauf, Bertelsmann Verlag, Bundesdrucksache 17/ 11465, S.157.

12 Circulaire de rentrée : la refondation de l'Ecole est en marche. Communiqué de presse Vincent Peillon - George Pau-Langevin, 11/04/2013, Ministère de l'éducation nationale http://www. education.gouv.fr; Circulaire d'orientation et de préparation de la rentrée 2013; NOR : MENE 1309444C circulaire n²013-060 du 10-4-2013, MEN - DGESCO Kultur- und Erziehungsministerium.

Ibid. Der hohe Stellenwert der kulturellen Bildung für die aktuelle französische Regierung ist erfreulich. Dabei sollte aber nicht vergessen werden, dass sie für Präsident Jacques Chirac ebenfalls wichtig war. Sein Premierminister Dominique de Villepin sprach ihr 2005 „politische Priorität“ zu aufgrund ihres wertvollen Beitrags zur Persönlichkeitsbildung und zur Bildung des Staatsbürgers. Cf. Jean-François Chaintreau, Culture et recherche $\mathrm{n}^{\circ} 106-$ 107, numéro spécial, Dezember 2005, S.57. 
befähigen.“ Der Bericht präzisiert, dass „,in einer Welt, deren soziale, politische und ökonomische Prozesse von einer Fülle ästhetischer Medien geprägt werden, [...] kulturelle/musisch-ästhetische Bildung zu einer wichtigen Voraussetzung für autonome und kritische Teilhabe an Gesellschaft und Politik“ wird. ${ }^{15}$ Als wesentlich gilt zudem Folgendes: „Die Begegnung und Auseinandersetzung mit Kunst und Kultur sind für jeden Menschen von prägender Bedeutung. Sie beeinflussen die sinnliche Wahrnehmung, die kreativen Fertigkeiten und die Ausdrucksfähigkeit. Sie ermöglichen einen Zugang zur Geschichte, zu den Traditionen, Werten und kulturellen Leistungen in Deutschland, Europa und der Welt. Durch kulturelle Bildung werden wichtige Grundlagen für den gesellschaftlichen Zusammenhalt geschaffen." ${ }^{\text {"16 }}$ Diese Beispiele zeigen, dass zwischen Deutschland und Frankreich in wesentlichen Punkten Übereinstimmung besteht. Gleichwohl können wir feststellen, dass der Aspekt Persönlichkeitsbildung in Deutschland stärker hervorgehoben wird als in Frankreich, was wohl darauf zurückgeführt werden kann, dass hier der humanitätsphilosophische Bildungsbegriff der deutschen Klassik unterschwellig weiter wirkt. Auf Landesebene schreibt zum Beispiel Stefan Krimm, Ministerialrat im Bayerischen Staatsministerium für Unterricht und Kultus: „Eine wesentliche Dimension ästhetischer Bildung ist aber auch ihr Beitrag zur Persönlichkeitsbildung. ${ }^{\text {“17 }}$

Des Weiteren fällt auf, dass in Frankreich sogenannte „TransferArgumente" sehr wichtig sind, also die Auffassung, dass Schüler durch Beschäftigung mit Kunst Kompetenzen erwerben, die für andere Bereiche wichtig sind: Gedächtnisschulung, Fähigkeit sich in der Öffentlichkeit zu produzieren, Teamgeist entwickeln, usw. In Deutschland kann man Folgendes lesen: „Es ist keineswegs so, dass Mozart, dass Musik irgendwie automatisch kognitiv „schlau“ macht. Und es Ist auch keineswegs so, dass Tanz und

15 Bildungsbericht der Bundesregierung, Kulturellel musisch-ästhetische Bildung im Lebenslauf, S.157; Bertelsmann Verlag, Bundesdrucksache 17/ 11465.

16 "Kultur und kulturelle Bildung vermitteln Traditionen, Kenntnisse und Werte, die eine Gesellschaft erst lebenswert machen", erklärt Kulturstaatsminister Bernd Neumann. Website der Bundesregierung, eingesehen am 4.Juni 2013.

17 Stefan Krimm, „Ästhetische Bildung - eine Zukunft voller Möglichkeiten“, Politische Studien $\mathrm{n}^{\circ} 385$, September/ Oktober 2002, S.25. 
Theater in jedem Fall sozial machen." ${ }^{18}$ Die aktuellen französischen Richtlinien zur künstlerisch-ästhetischen Bildung lassen wenig Raum für folgende Überlegung: „Die spezifische Begründung [kultureller Bildung] kann daher nur aus der Kultur, aus den Künsten selbst stammen: Sie bieten unvergleichliche Erfahrungs- und Bildungswelten, die das Leben über die gesamte Lebensspanne bereichern und die gerade durch die NichtAlltäglichkeit, ihre Differenz zum Alltag gekennzeichnet sind. “" ${ }^{\text {19 }}$

Was die Medienkompetenz betrifft, die auch in den Bereich der Erziehung durch Kultur gehört, so ist sie in Frankreich im Rahmen der NTIC („Nouvelles technologies d'information et de communication“) von großer Bedeutung. Es zeichnet sich aber ab, dass dabei weniger als in Deutschland ein verantwortungsvoller Umgang mit ihnen gelernt werden soll. Texte der Ständigen Konferenz der Kultusminister und der Bund-Länder-Kommission für Bildungsplanung und Forschungsförderung bestimmen eindeutig, durch Auswahl, Nutzung, Gestaltung, Analyse und Bewertung einen kritischen Umgang mit den neuen Medien zu ermöglichen. ${ }^{20}$ In Frankreich fordert das Rundschreiben vom 11.April 2013 zwar, die Anwendung der digitalen Medien in der Schule zu fördern, dem Text ist aber nicht zu entnehmen, dass ein kritischer Umgang mit ihnen zu lehren und zu üben sei. Unter der Überschrift „Faire entrer l'Ecole dans l'ère numérique“ (Die Schule ins digitale Zeitalter führen) ist vielmehr vorgesehen: „Die Schule [...] soll alle Schüler beim Erwerb und der Beherrschung digitaler Kompetenzen begleiten. Mit Hilfe digitaler Medien sollen auch ,attraktive, innovative und effiziente pädagogische Praxen“ entwickelt und damit das gesamte Schulsystem verbessert werden; mittelfristig geht es darum, „neue digitale Systeme aufzubauen". Es wird also besonderer Wert auf den Auf- beziehungsweise Ausbau der Kommunikationsnetze gelegt, wobei die Frage ihres verantwortungsbewussten Gebrauchs nicht direkt angesprochen wird. Wie

\footnotetext{
18 Eckart Liebau, „Kultur leben“, Impulse zur Bildungsdiskussion, Vierte Nürnberger Bildungskonferenz „Kulturelle Bildung im Lebenslauf“ am 17. Oktober 2012 im KunstKultur-Quartier Nürnberg, S.9.

19 Ibid., S. 9-10.

20 Cf. Christof Gramm, Schlüsselqualifikationen für Staatsbürger, Politik verstehen, Demokratie bejahen, Olzog Aktuell GmbH, 2010.
} 
wichtig dieser Aspekt ist, wurde allerdings bereits 2005 an anderer Stelle betont - im Kulturministerium. So verwendet Jean-François Chaintreau in seinem Plädoyer für eine Verstärkung der kulturellen Bildung - als „Faktor des Respekts vor der kulturellen Vielfalt und der Identität jedes Einzelnen“ auch folgendes Argument: Sie ist ein Gegengewicht zur Überschwemmung mit Informationen durch neue Kommunikationstechnologien. ${ }^{\text {"21 }}$

\section{Intellekt, Emotivität, Imagination, Expressivität}

In Rundschreiben, Berichten und Kommentaren zur Bildungspolitik in Deutschland und Frankreich zeichnet sich ein gewisser Unterschied zwischen beiden Ländern ab in Bezug auf den jeweiligen Stellenwert von Intellekt, Emotivität, Imagination und Expressivität. In Deutschland ist kulturelle Bildung stärker mit dem Wunsch nach Förderung der Ausdrucksfähigkeit verbunden, die Entwicklung der emotiven und imaginativen Anlagen des Kindes erhält mehr Aufmerksamkeit als in Frankreich. Besonders deutlich wird dies auf Landesebene im Freistaat Bayern, wo Artikel 131 der Verfassung in Absatz 1 festlegt: "Die Schulen sollen nicht nur Wissen und Können vermitteln, sondern auch Herz und Charakter bilden." ${ }^{\text {"22 }}$ In Frankreich ist in Anschluss an Descartes und die rationalistische Tradition der Aufklärung das Interesse für die Schulung des Intellekts grösser. ${ }^{23}$ So dass auch im Bereich der kulturellen Bildung die Förderung von Emotivität und Ausdrucksfähigkeit weniger Aufmerksamkeit erhält als in Deutschland. Der hohe Stellenwert der intellektuellen Schulung in Frankreich fällt zum Beispiel auf, wenn es darum geht, Musik an Schulen zu rechtfertigen. So finden wir im Rundschreiben vom 11.April 2013 als Begründung nicht nur, dass Musik oft für Kinder die erste Gelegenheit ist, sich in der Öffentlichkeit zu produzieren, dass sie Teamgeist, Integration und sozialen Zusammenhalt fördert und zur Entdeckung des kulturellen Erbes beiträgt. Es wird auch darauf hingewiesen, dass Singen die Allgemeinbildung fördert und das Gedächtnis der Schüler stärkt. Womit man sich die Frage stellen kann, inwieweit Diderots Kritik an

\footnotetext{
$21 \quad$ Jean-François Chaintreau, op.cit.

$22 \quad$ Verfassung des Freistaates Bayern vom 2.Dezember 1946.

23 education.gouv.fr, De la maternelle au baccalauréat-l'éducation artistique et culturelle.
} 
der Musikpraxis des 18. Jahrhunderts noch eine gewisse Aktualität hat im Diderotjahr 2013 anlässlich des 300. Geburtstages des Philosophen. In der „Klavierschule des Herrn Bemetzrieder“ kritisiert Diderot nämlich Klavierspieler, welche ohne Gefühl spielen und er distanziert sich von jenen Komponisten, die er „compositeurs de mémoires“ nennt, weil sie zu intellektuell vorgehen und ,aus dem Gedächtnis Fetzen zusammenflicken, die sie bei anderen Komponisten gefunden haben “ ${ }^{24}$

\section{Gleichheit und Diversität}

Wie lässt sich der hohe Stellenwert des Intellekts in der künstlerischkulturellen Bildung in Frankreich erklären? Neben dem Nachwirken Descartes und der rationalistischen Tradition der Aufklärung kann an eine weitere Erklärungsmöglichkeit gedacht werden. Man könnte diese große Kopflastigkeit auch darauf zurückführen, dass das Prinzip der Gleichheit in Frankreich von ganz besonderer Bedeutung ist. Wer Gleichheit sagt, denkt auch an die Möglichkeit eines Vergleichs, was wiederum bedeutet: Überprüfbarkeit, ja Messbarkeit der Leistungen und Ergebnisse. Und dies ist tatsächlich auf intellektuellem Gebiet leichter als auf der affektiv-imaginativen Ebene. So ließe sich erklären, dass in Frankreich großer Wert auf die Vermittlung von Kunstgeschichte gelegt wird, die in entsprechenden deutschen Texten nicht eigens erwähnt wird. Denn kunsthistorische Kenntnisse lassen sich leichter abfragen und damit vergleichen als die Ausdrucksfähigkeit und Kreativität der Schüler.

Die obersten Werte der französischen Republik Freiheit, Gleichheit, Brüderlichkeit und insbesondere das Gleichheitsprinzip könnten also durchaus ein Grund dafür sein, dass manche Lernziele, die in Deutschland ganz oben stehen, in Frankreich weniger wichtig sind. Und dem Ideal der französischen Republik entsprechend - Artikel 1 der Verfassung vom 4. Oktober 1985 spricht von der „unteilbaren Republik“- finden wir in den offiziellen Texten folgenden Aspekt praktisch nicht, der in Deutschland große Bedeutung hat. Ich meine die Interkulturalität. Im Rundschreiben vom 11. April 2013 kommt das Wort „interkulturell“ nicht vor. Das Prinzip der republikanischen

24 Denis Diderot, Leçons de clavecin et principes d'harmonie par M.Bemetzrieder, Ouevres de Diderot, AT, XII, 186. 
Gleichheit sowie das Bestreben nach möglichst großer Einheit - „la République une et indivisible" - führt also auch dazu, dass die Öffnung auf Interkulturalität in Frankreichs Schulprogrammen weniger ausgeprägt ist als in Deutschland. Folgende Beobachtung aus dem Jahre 2002 ist im Wesentlichen auch heute noch aktuell, zumindest auf Regierungsebene hat sich diesbezüglich nichts geändert: „In Frankreich tendiert die republikanische Ideologie dazu, die Pluralität der Kulturen als ein Hindernis für die Gleichheit zu behandeln, besonders wenn sie in der Öffentlichkeit in Erscheinung tritt. Dies erklärt, dass die Frage des Interkulturellen aus den offiziellen Instruktionen des Erziehungsministeriums ausgeschlossen wird." ${ }^{25}$

Zum Abschluss ist zu sagen: Der Wert der ästhetischen Erziehung wird sowohl in Deutschland als auch in Frankreich anerkannt und folgende Aussage der Bundeszentrale für politische Bildung könnte auch in einem französischen Text stehen: „Kunst und Kultur sind Motoren gesellschaftlicher Entwicklungen - das macht kulturelle Bildung so bedeutsam." ${ }^{\text {"26 }}$ Dies schließt jedoch nicht aus, dass sie in beiden Ländern teilweise unter anderen Vorzeichen geschieht. Und dass die entsprechende Praxis politische Prinzipien ebenso wie Denk- und Bildungstraditionen reflektiert. Descartes ist eben in Frankreich stärker verwurzelt als Schiller - obwohl die französische Nationalversammlung im Oktober 1792 dem Verfasser der Briefe über die ästhetische Erziehung des Menschen das französische Bürgerrecht verlieh. Dies sollte im deutsch-französischen Jahr 2013 aus Anlass des 50. Jahrestages des Elysée-Vertrags über die deutsch-französische Zusammenarbeit und auch in Zukunft als Anregung dienen, Gemeinsamkeiten und Unterschiede zwischen beiden Ländern weiter zu erforschen -zum besseren Verständnis der jeweiligen Bildungskultur.

25 Françoise Lorcerie, VEI-Enjeux (Ville-Ecole-Intégration) n ${ }^{129}$, Juni 2002, S.170.

26 Bundeszentrale für politische Bildung: http://www.bpb.de, eingesehen am 4.6.2013. 


\section{Literaturverzeichnis}

BILDUNGSBERICHT DER BUNDESREGIERUNG (2012): Autorengruppe Bildungsberichterstattung, Bildung in Deutschland 2012. Ein indikatorengestützter Bericht mit einer Analyse zur kulturellen Bildung im Lebenslauf, gefördert mit Mitteln der Ständigen Konferenz der Kultusminister der Länder in der Bundesrepublik Deutschland und des Bundesministeriums für Bildung und Forschung, hrsg. Bundesministerium für Bildung und Forschung, W. Bertelsmann Verlag, Bielefeld 2012, 360S.; Bildungsbericht der Bundesregierung, Kulturelle/ musisch-ästhetische Bildung im Lebenslauf; Bertelsmann Verlag, Bundesdrucksache 17/ 11465.

BUNDESZENTRALE FÜR POLITISCHE BILDUNG (2013) http://www.bpb.de, eingesehen am 4.6.2013.

CHAINTREAU, Jean-François (2005): «Education artistique et culturelle, une priorité politique », Culture et recherche n ${ }^{\circ} 106-107$, numéro spécial, décembre 2005

DIDEROT, Denis (1771), Leçons de clavecin et principes d'harmonie par M.Bemetzrieder. In Euvres complètes, éd. Assézat-Tourneux, Paris, 1875-1877

FRANZÖSISCHES ERZIEHUNGSMINISTERIUM (2013) : PEILLON, Vincent/ PAULANGEVIN, George (2013) : Communiqué de presse, Circulaire de rentrée : la refondation de l'Ecole est en marche. 11/04/2013, Ministère de l'éducation nationale http://www.education.gouv.fr; Circulaire d'orientation et de préparation de la rentrée 2013 ; NOR: MENE 1309444C circulaire n²013-060 du 10-4-2013, MEN - DGESCO; education.gouv.fr, De la maternelle au baccalauréat - l'éducation artistique et culturelle

FREISTAAT BAYERN (1946): Verfassung des Freistaates Bayern vom 2.Dezember 1946.

GRAMM, Christof (2010): Schlüsselqualifikationen für Staatsbürger. Politik verstehen, Demokratie bejahen, Olzog Aktuell GmbH, 2010.

KRIMM, Stefan (sd): „Ästhetische Bildung - ein Konzept nicht nur für Gymnasien“. In Bayerisches Staatsministerium für Unterricht und Kultus, Ästhetische Bildung. Handreichung, hrsg. vom Staatsinstitut für Schulqualität und Bildungsforschung, München, sd.

KRIMM, Stefan (2002): „Ästhetische Bildung - eine Zukunft voller Möglichkeiten“, Politische Studien $\mathrm{n}^{\circ} 385$, September/ Oktober 2002.

LIEBAU, Eckart (2012): „Kultur leben“, Impulse zur Bildungsdiskussion, Vierte Nürnberger Bildungskonferenz „Kulturelle Bildung im Lebenslauf“ am 17. Oktober 2012 im KunstKultur-Quartier Nürnberg.

LORCERIE, Françoise (2002) : VEI-Enjeux (Ville-Ecole-Intégration) n¹29, juin 2002.

SCHILLER, Friedrich (1793-94): Briefe über die ästhetische Erziehung des Menschen. In Friedrich Schiller, Ausgewählte Werke, hrsg. und eingeleitet von Ernst Müller, Bd.5 Schriften zum Theater, zur Philosophie der Ästhetik und der Geschichte, Kohlhammer Verlag Stuttgart, 1955, S. 336.

STÄNDIGE KONFERENZ DER KULTUSMINISTER DER LÄNDER IN DER BUNDESREPUBLIK DEUTSCHLAND (2012): Aufstieg durch Bildung, Die Qualifizierungsinitiative für Deutschland, Bericht zur Umsetzung 2012, Sekretariat der 
Ständigen Konferenz der Kultusminister der Länder in der Bundesrepublik Deutschland; Gemeinsame Wissenschaftskonferenz (GWK), Beschluss der KMK vom 18.10.2012, Beschluss der GWK vom 16.11.2012. Abrufbar unter: www.kmk.org sowie www.gwkbonn.de und www.bmbf.de.

SCHOBER, Angelika (2013): „Schillers Briefe über die ästhetische Erziehung des Menschen als Antwort auf die Französische Revolution und ihre Folgen“, Kairoer Germanistische Studien $\mathrm{n}^{\circ} 20,2012-2013, \mathrm{~S} .213-233$.

UNESCO (2006): Leitfaden für kulturelle Bildung. Schaffung kreativer Kapazitäten für das 21. Jahrhundert, Paris, 2006. 